\title{
Waveguide Raman Spectroscopy of Thin Polymer Layers and Monolayers of Biomolecules Using High Refractive Index Waveguides
}

\author{
Johannes Sake Kanger, Cees Otto, Michiel Slotboom, and Jan Greve* \\ Applied Optics Group, Department of Applied Physics, BMTI and MESA Research Institutes, \\ University of Twente, P.O. Box 217, 7500 AE Enschede, The Netherlands
}

Received: August 31, 1995; In Final Form: November 21, $1995^{\circledR}$

\begin{abstract}
We demonstrate the use of a high refractive index waveguide structure for waveguide Raman experiments to detect thin (mono)layers of biological macromolecules on top of these waveguides. Theoretically, a combination of a thin, high refractive index layer on top of a low index substrate results in an increase in

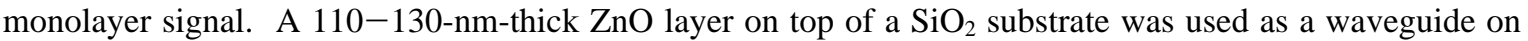
which a very thin (8-nm) polystyrene layer could be detected with a signal-to-noise ratio $(S / N)$ of $\sim 60$ for a 60 -s integration time. With this waveguide, Raman spectra of a monolayer of the protein bovine albumin and of a dimyristoylphosphatidylethanolamine (DMPE) Langmuir-Blodgett monolayer were measured. To our knowledge, this is the first time a waveguide Raman spectrum of a monolayer of protein is reported without resonant enhancement techniques.
\end{abstract}

\section{Introduction}

Waveguide Raman spectroscopy (WRS) has proved to be a useful technique to study thin layers of molecules. ${ }^{1}$ The pump laser beam is coupled into a planar dielectric waveguide in order to probe the Raman-active vibrations of the waveguiding material. Due to the small dimensions of the waveguide, which are typically a micrometer in thickness, large electric field strengths can be obtained in the waveguide over centimeter distances. The combination of high intensities combined with a large sampling volume gives rise to an increase in Raman signal of 3-4 orders ${ }^{1}$ of magnitude compared to conventional Raman scattering geometries. Spectra of polymer waveguides with thicknesses of a few micrometers were first reported by Levy et al. ${ }^{2}$ When, however, the thickness of the layers becomes smaller than a few $100 \mathrm{~nm}$, the waveguide can no longer guide the light. Therefore, another approach is necessary to obtain Raman spectra of very thin layers. If the thin layer is deposited on a "thick" waveguide, the evanescent field can be used to probe the Raman-active vibrations of the thin layer. ${ }^{3}$ In this way, there is no limitation to the thickness of the thin layer. Using this method, Raman spectra of 80-nm thin polystyrene layers were measured on a Corning 7059 waveguide. ${ }^{3}$ Later, also Raman spectra of nine monolayers of cadmium arachidate (CdA) could be detected. ${ }^{4,5}$ Also, resonant enhancement techniques can be used in combination with WRS. Monolayers of porphyrins, ${ }^{6}$ cytochrome $c{ }^{7}$ and cyanine ${ }^{3,8}$ on top of a waveguide resulted in resonant Raman spectra with good signalto-noise ratios.

It is evident that an intense evanescent field is important in obtaining large signal levels. The evanescent field is strongly influenced by the thickness and refractive indices of the waveguide. Different waveguides composed of Corning $7059,{ }^{3-5,8,9} \mathrm{SiO}_{x} \mathrm{~N}_{y},{ }^{7}$ and polymer (polystyrene ${ }^{6,9}$ ) film layers are used for WRS of thin layers. All those waveguides have a low refractive index, (1.5-1.6), and they are used with a thickness of $1 \mu \mathrm{m}$ or more. We will show that a higher refractive index and a smaller thickness of the waveguide can result in a signal level increase of 1 order of magnitude with

* Author to whom correspondence should be addressed.

${ }^{\otimes}$ Abstract published in Advance ACS Abstracts, February 1, 1996.

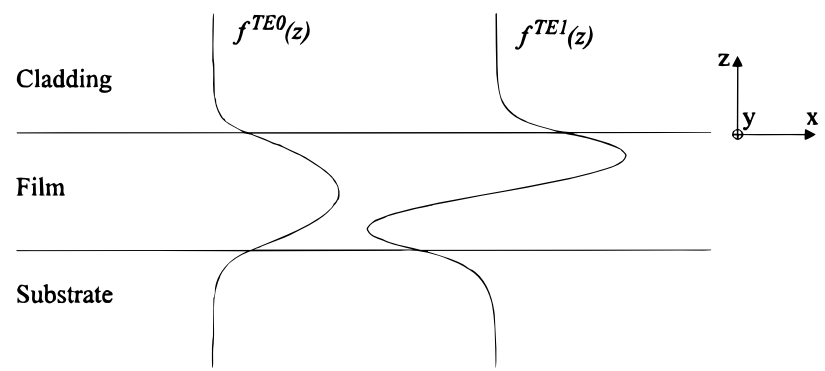

Figure 1. Schematic representation of the waveguide geometry as used for the calculations. The electric field distribution functions are shown for the TE0 and TE1 mode and were calculated for a 500-nm-thick film with a refractive index of 2.0 on a substrate with a refractive index of 1.46. The refractive index of the cladding is 1 , and the wavelength is $514.5 \mathrm{~nm}$.

similar pump powers. Using small thickness waveguides does not only result in a higher evanescent field but also in an increase of the signal-to-background ratio. This background, originating from Raman and/or fluorescence of the waveguide, can obscure the signal from the thin layer. We have used a $\mathrm{ZnO}$ waveguide, which has a refractive index of 2.0. Because of this high refractive index, the thickness of the waveguide can be as small as $100 \mathrm{~nm}$. With this waveguide, it was possible to detect very thin layers of polystyrene $(8 \mathrm{~nm})$ and also monolayers of the protein albumin and of a single Langmuir-Blodgett layer using a pump power of only $5 \mathrm{~mW}$ in the waveguide. To our knowledge, this is the first time a spontaneous Raman spectrum of a monolayer of a protein is presented. Raman spectra of Langmuir-Blodgett monolayers have been published in the literature using reflection spectroscopy, ${ }^{10,11}$ a Raman microscope combined with internal reflection, ${ }^{12}$ and surface plasmon enhanced techniques. ${ }^{13,14}$ However, the spectrum presented in this paper is the first obtained with spontaneous WRS.

\section{Theory}

The waveguide considered here (Figure 1) consists of three dielectric layers: cladding (air), film ( $\mathrm{ZnO}$ ), and substrate $\left(\mathrm{SiO}_{2}\right)$. If the refractive index of the film layer is higher than the refractive index of both cladding and substrate, light can be guided by the waveguide. Waveguides only contain a limited 
number of guiding modes, with two different polarizations, TM modes which are polarized perpendicular to the $y$ direction and TE modes which are polarized parallel to the $y$ direction. The electric field of a guiding mode is not completely confined to the film layer but penetrates into the cladding and substrate. This part of the electric field is called the evanescent field. At the boundary between the cladding and the film, a thin layer of molecules is deposited. Evidently the Raman signal strength from the thin layer will be proportional to the intensity of the pump mode at this interface. The electric field distribution of a guided TE mode in a waveguide can be written as

$$
\vec{E}^{\mathrm{TE} m}(z, x, \omega)={ }_{1}{ }_{2} a^{\mathrm{TE} m} \hat{e}_{y} f^{\mathrm{TE} m}(z) \exp \left\{i\left(\omega t-\beta^{\mathrm{TE} m} x\right)\right\}+\mathrm{cc}
$$

For a TM mode, it is more convenient to write down the expression for the magnetic field:

$$
\vec{H}^{\mathrm{TM} m}(z, x, \omega)={ }_{1} /{ }_{2} a^{\mathrm{TM} m} \hat{e}_{y} f^{\mathrm{TM} m}(z) \exp \left\{i\left(\omega t-\beta^{\mathrm{TM} m} x\right)\right\}+\mathrm{cc}
$$

The discrete modes are enumerated by $m(m=0,1,2, \ldots)$. In these formulas, $f^{\mathrm{TE} m}(z)$ and $f^{\mathrm{TM} m}(z)$ denote the electric field distribution functions for the TE and TM modes, $a^{\mathrm{TE} m}$ and $a^{\mathrm{TM} m}$ the amplitudes of the modes, $\beta^{\mathrm{TE} m}$ and $\beta^{\mathrm{TM} m}$ the mode propagation vectors, and $\omega$ the frequency of the propagating light. The electric field distribution functions $f^{\mathrm{TE} m}(z)$ and $f^{\mathrm{TM} m}(z)$ are normalized such that $\left|a^{m}\right|^{2}$ gives the guided power of the mode per meter of wavefront (in the $y$ direction). There are several ways to calculate the electric field distribution functions and the corresponding propagation vectors of a three-layer waveguide. We used the matrix method given by Ghatak et al. ${ }^{15}$ As an example, the electric field distribution functions $f^{\mathrm{TE} 0}(z)$ and $f^{\mathrm{TE} 1}(z)$ are shown in Figure 1.

The intensity at the interface between cladding and film $(z$ $=0)$ is given by

$$
\begin{aligned}
& I_{\text {surface }}^{\mathrm{TE} m}={ }^{1} /{ }_{2} \operatorname{Re}\left[\vec{E}^{\mathrm{TE} m}(z=0) \times \vec{H}^{\mathrm{TE} m_{*}}(z=0)\right]= \\
& { }_{1}{ }_{2} N_{\mathrm{eff}} \epsilon_{0} c\left|a^{\mathrm{TE} m}\right|^{2}\left(f^{\mathrm{TE} m}(z=0)\right)^{2}
\end{aligned}
$$

for TE modes and

$$
\begin{aligned}
I_{\text {surface }}^{\mathrm{TM} n}={ }_{1}^{1} \operatorname{Re}\left[\vec{E}^{\mathrm{TM} m}(z=0) \times \vec{H}^{\mathrm{TM} m} *(z=0)\right]= \\
1{ }_{2} N_{\mathrm{eff}} n_{\mathrm{c}}{ }^{-2} \mu_{0} c\left|a^{\mathrm{TM} m}\right|^{2}\left(f^{\mathrm{TM} m}(z=0)\right)^{2}
\end{aligned}
$$

for TM modes. In these equations, $n_{\mathrm{c}}$ denotes the refractive index of the cladding and $N_{\text {eff }}$ is the effective refractive index of the specific mode defined as $N_{\text {eff }}=\beta c / \omega$. Due to the normalization, we can write for $\left|a^{\mathrm{TE} m, \mathrm{TM} m}\right|^{2}$

$$
\left|a^{\mathrm{TE} m, \mathrm{TM} m}\right|^{2} H=P
$$

where $H$ is the beam width inside the waveguide and $P$ is the power in the mode. By combining eqs 3 and 5, we find for the intensity at the surface for TE modes

$$
I_{\text {surface }}^{\mathrm{TE} m}=(P / H)(1 / 2) N_{\text {eff }} \epsilon_{0} c\left(f^{\mathrm{TE} m}(z=0)\right)^{2}=(P / H) I_{\text {norm }}^{\mathrm{TE} m}
$$

and with substituting eq 4 into eq 5 , a similar expression is obtained for TM modes:

$$
I_{\text {surface }}^{\mathrm{TM} m}=(P / H)(1 / 2) N_{\mathrm{eff}} n_{\mathrm{c}}{ }^{-2} \mu_{0} c\left(f^{\mathrm{TM} m}(z=0)\right)^{2}=(P / H) I_{\mathrm{norm}}^{\mathrm{TM} m}
$$

We have introduced the normalized intensities $I_{\text {norm }}^{\mathrm{TE} m}$ and $I_{\text {norm }}^{\mathrm{TM} m}$ to eliminate the experimental details about pump power and

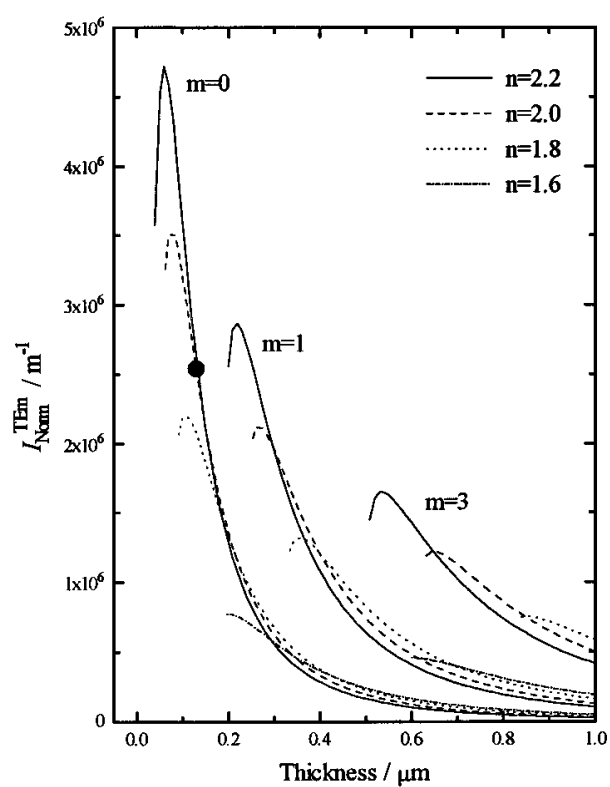

Figure 2. Normalized surface intensity $I_{\text {norm }}^{\mathrm{TEm}}$ as function of the film layer thickness for different modes $(m=0,1$, and 3$)$ and for different values of the refractive index $n$ of the film layer. The black dot indicates the waveguides used for measurements on the polymer and protein layers.

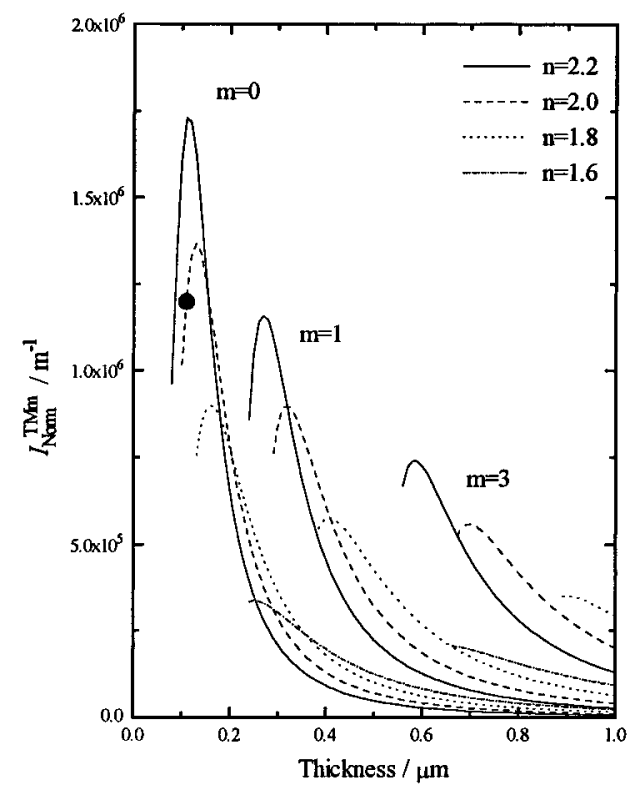

Figure 3. Normalized surface intensity $I_{\text {norm }}^{\mathrm{TM} m}$ as function of the film layer thickness for different modes ( $m=0,1$, and 3) and for different values of the refractive index $n$ of the film layer. The black dot indicates the waveguides used for measurements on Langmuir-Blodgett layers.

beam widths inside the waveguide. These normalized intensities depend only on the details of the waveguide structure and have units of $\mathrm{m}^{-1}$. We have analyzed the normalized intensities as a function of refractive index and thickness of the film layer for different modes $m$. For the substrate, the refractive index of $\mathrm{SiO}_{2}$ was chosen $(n=1.46)$. The wavelength was fixed at $514.5 \mathrm{~nm}$. The results are shown in Figure 2 for the TE modes and in Figure 3 for the TM modes. The dependences of the normalized intensities on the film layer thickness are given for three different modes ( $m=0,1$, and 3 ). For each mode, four different refractive indices for the film layer were analyzed. For a given refractive index, the intensity reaches a maximum at a thickness which is slightly higher than the cut-off thickness. Below the cut-off thickness, the mode can no longer be guided 
by the waveguide. The origin of the optimum in the thickness can be explained as follows. In the case of a thick layer, most of the light is confined to the guiding layer. When the thickness is decreasing, the light is still confined to the layer, and consequently, the intensity increases. However, when the thickness approaches the cut-off thickness, the evanescent field in the substrate becomes very large and the light is no longer concentrated in the film layer. As a result, the intensity drops just before the cut-off thickness. For high refractive index layers, the cut-off thickness is smaller, leading therefore to higher intensities at the surface for small thicknesses. This behavior can be clearly seen in Figure 2 for all the TE as well as for all the TM modes (Figure 3). The black dots which are plotted in the figure indicate the waveguide structures which are used in the experiments described in this paper. Compared to the $1-\mu \mathrm{m}$-thick Corning $7059(n=1.56)$ waveguides, ${ }^{3-5,8,9}$ there is an order of magnitude increase in the surface intensity. Consequently, this will lead to a monolayer signal increase with an order of magnitude at similar pump powers.

\section{Experimental Section}

An 1800-nm-thick $\mathrm{SiO}_{2}$ substrate was obtained by wet oxidation of a 3 -in. Si wafer at $1150{ }^{\circ} \mathrm{C}$ for $11 \mathrm{~h}$. On top of this substrate, a $\mathrm{ZnO}$ film layer was fabricated in an rf magnetron sputtering plant. An rf power of $1800 \mathrm{~W}$, an oxygen pressure of $4.0 \times 10^{-4} \mathrm{mbar}$, and a substrate temperature of $590{ }^{\circ} \mathrm{C}$ were used and resulted in a low loss $(<2 \mathrm{~dB} / \mathrm{cm})$ waveguide. In this way, several waveguides with thicknesses ranging from 110 to $130 \mathrm{~nm}$ and with a refractive index of 1.98 for TE modes and 2.00 for TM modes were made. The thickness and refractive indices of the waveguides were determined experimentally by fitting the measured coupling angles for the TE0 and TM0 modes using the thickness and refractive index as the fitting parameters. The waveguides were stored in a box flushed with nitrogen to prevent the degradation of the $\mathrm{ZnO}$ layer in the atmosphere.

A solution of polystyrene dissolved in toluene $(0.5 \% \mathrm{wt})$ was spin coated at $4000 \mathrm{rpm}$ for $20 \mathrm{~s}$ on a 130-nm-thick $\mathrm{ZnO}$ waveguide. This resulted in a 8-nm-thick polystyrene layer as measured by a Dektak surface profiler and corresponds to the expected thickness based on the polystyrene concentration. ${ }^{16}$

The protein bovine albumin (BA) was purchased from Sigma (No. A7906) and used without further purification. A 130-nmthick $\mathrm{ZnO}$ waveguide was dipped in a $10^{-5} \mathrm{M}$ BA in phosphatebuffered saline (PBS) and left in the solution for about $30 \mathrm{~min}$. The waveguide was then rinsed several times with ultrapure water to remove any nonadsorbed proteins. This procedure is known to result in a surface density of approximately $2 \mathrm{mg} /$ $\mathrm{m}^{2},{ }^{17,18}$ which in the case of the protein BA corresponds more or less to a monolayer coverage. ${ }^{17}$

Langmuir-Blodgett (LB) layers were obtained by spreading a $1 \mathrm{mg} / \mathrm{mL}$ solution of the lipid dimyristoylphosphatidylethanolamine (DMPE) in 3:1 (v/v) chloroform/methanol onto the subphase (deionized water, $\mathrm{pH} \sim 7.5$ ). The monolayer was transferred to a 110 -nm-thick $\mathrm{ZnO}$ waveguide, with the hydrophilic headgroup at the surface, at a surface pressure of $23 \mathrm{mN} / \mathrm{m}$ and a molecular area of $0.6 \mathrm{~nm}^{2}$, which corresponds to the liquid condensed phase; the transfer ratio was $\sim 1$. The thickness of the monolayer was $2.5 \mathrm{~nm}$.

The waveguide Raman spectrometer (Figure 4) consisted of an $\mathrm{Ar}^{+}$ion laser, a coupling device, a spectrometer, and a liquid $\mathrm{N}_{2}$ cooled CCD camera. The light of the $\mathrm{Ar}^{+}$ion laser, operated at $514.5 \mathrm{~nm}$, was coupled into a single mode fiber. This fiber directed the light to a waveguide coupling device. In this way, the position of the waveguide can be adjusted using an $x-y$

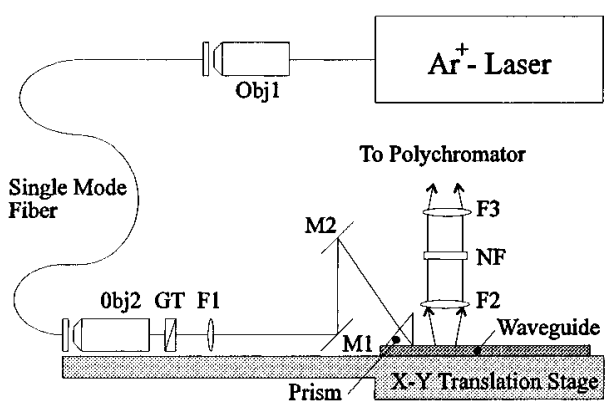

Figure 4. Waveguide Raman setup. Obj, microscope objective, M, mirror; GT, Glan Thompson polarizor; F1, 200-mm lens; F2, 35-mm lens; F3, 100-mm lens; NF, holographic notch filter for $514.5 \mathrm{~nm}$. Obj2, GT, F1, M1, M2, Prism, and waveguide are all fixed to the $x-y$ translation stage.

translation stage for alignment purposes without perturbing the coupling into the waveguide. The waveguide coupling device consisted of a 200-mm lens (F1) which focused the light via a set of two mirrors (M1, M2) onto the prism base of a $\mathrm{SrTiO}_{3}$ $(n=2.46$ at $514.5 \mathrm{~nm}$ ) prism which was used for coupling the light into the waveguide. The two mirrors were used to adjust the proper coupling angle for the desired modes. For all the experiments presented here, the power of the laser beam at the base of the prism was $50 \mathrm{~mW}$. The coupling efficiency was estimated to be approximately $10 \%$, resulting in a power of 5 $\mathrm{mW}$ inside the waveguide. This results in a surface intensity of $\sim 2 \times 10^{8} \mathrm{~W} / \mathrm{m}^{2}$. About 5-mm length of the 50- $\mu \mathrm{m}$-wide strike of light in the waveguide was imaged on the entrance slit of a Yobin-Yvon HR460 spectrometer using a set of two lenses (F2, F3) with a magnification of 3 and a collection numerical aperture (NA) of 0.4. A holographic notch filter was used to reject the stray light. The spectra were recorded with a Tektronix thinned back-illuminated CCD camera mounted in a liquid $\mathrm{N}_{2}$ filled Dewar which cooled the detector down to $-120{ }^{\circ} \mathrm{C}$.

\section{Results and Discussion}

The spectrum obtained from the 8-nm polystyrene layer is depicted in Figure 5. The spectrum was recorded with a spectral resolution of $2.6 \mathrm{~cm}^{-1} /$ pixel in $60 \mathrm{~s}$ and a TE0 excitation mode. The strong band at $1156 \mathrm{~cm}^{-1}$ (denoted with an asterisk) originates from the $\mathrm{ZnO}$ layer. This band may hamper the detection of weak signals in this spectral range. All other vibrations in the spectrum can be assigned to the thin polystyrene layer. There is another strong band of $\mathrm{ZnO}$ at $440 \mathrm{~cm}^{-1}$, but since it does not interfere with the thin-layer spectrum, it is not shown in the figure. A $S / N$ of $\sim 60$ is found for the strong bands around $3000 \mathrm{~cm}^{-1}$, and for the much weaker bands in the 1600$\mathrm{cm}^{-1}$ region, a $S / N$ of $\sim 10$ is found. This result shows that Raman spectra of very thin layers of polymers can be measured with a good $S / N$, and it is anticipated that Raman spectra of monolayers of polymers should be easily obtainable.

The Raman spectrum of a monolayer of BA is given in Figure 6. The integration time needed to record this spectrum was $120 \mathrm{~s}$. The spectral resolution is $2.6 \mathrm{~cm}^{-1} /$ pixel. The measured wavenumber range extends from 2700 to $3150 \mathrm{~cm}^{-1}$. Most of the background of the waveguide was subtracted. The maximum signal level of the protein was about 6 counts/s, and the background from the waveguide was approximately 38 counts/ s. The integration time needed to record this spectrum with a $S / N$ of $\sim 10$ is short compared with the time to adsorb a full monolayer of protein, which is about $30 \mathrm{~min}$. This illustrates the possibility to study the adsorption kinetics of proteins. The $\mathrm{C}-\mathrm{H}$ aliphatic stretch at 2877 and $2931 \mathrm{~cm}^{-1} 19$ and the $\mathrm{C}-\mathrm{H}$ 


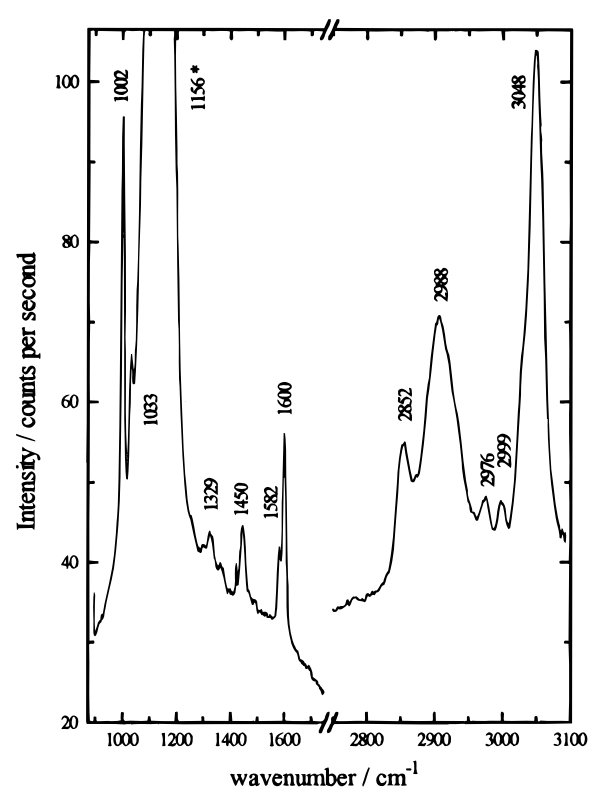

Figure 5. WRS spectrum of an 8-nm-thick polystyrene layer on top of a 130 -nm-thick $\mathrm{ZnO}$ waveguide. The strong $\mathrm{ZnO}$ optical phonon at $1156 \mathrm{~cm}^{-1}$ is indicated by an asterisk (*). All other bands originate from the thin polystyrene layer.

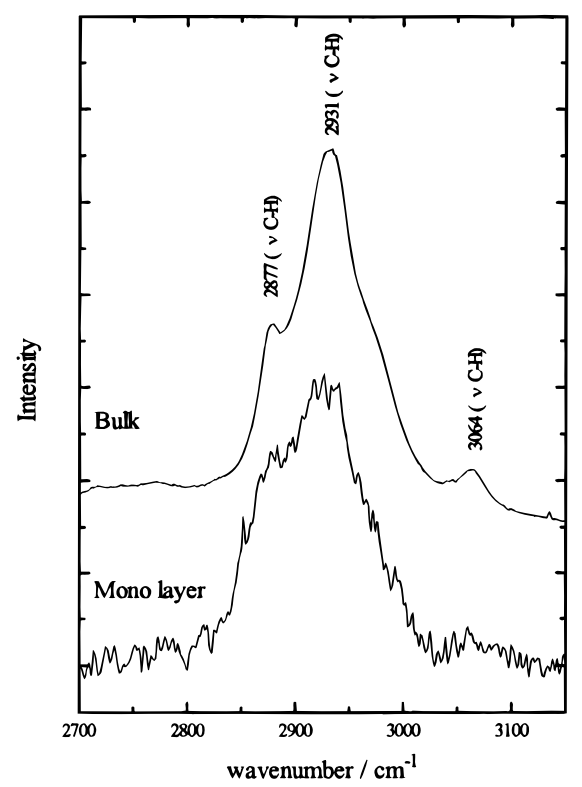

Figure 6. WRS spectrum of a monolayer of bovine albumin on top of a 130-nm-thick $\mathrm{ZnO}$ waveguide in the $\mathrm{C}-\mathrm{H}$ stretch range compared with the spectrum of bulk albumin. The vibrations are all indicated by their energies in wavenumbers.

aromatic stretch at $3064 \mathrm{~cm}^{-11} 19$ are clearly visible, but also the $2962-\mathrm{cm}^{-1}$ vibration ${ }^{20}$ can be spotted as a small shoulder. For comparison, we measured the spectrum of bulk BA. This spectrum is also shown in Figure 6 and is shifted vertically for convenience. After comparison of the two spectra, it can be concluded that the intensity of the aliphatic stretch vibration at $2931 \mathrm{~cm}^{-1}$ is lower compared with the other vibrations in the monolayer spectrum. The peak intensity ratio $I_{2931} / I_{2877}$ equals 2.1 for the bulk and 1.3 for the monolayer spectrum. Implications of the $\mathrm{C}-\mathrm{H}$ vibrations on the protein structure have not been extensively studied. However, there is an indication that the $\mathrm{C}-\mathrm{H}$ vibration of the $\mathrm{CH}_{3}$ group at $2931 \mathrm{~cm}^{-1}$ may have structural implications. ${ }^{21}$ A large increase in the Raman intensity at $2931 \mathrm{~cm}^{-1}$ is produced by the unfolding of RNase. It is suggested ${ }^{21}$ that this is caused by the exposure of previously buried aliphatic amino acid residues to the surrounding water.

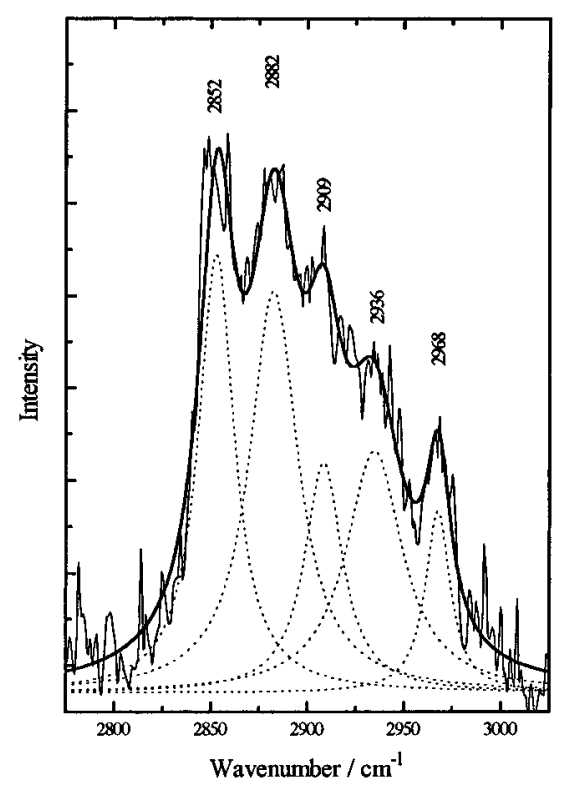

Figure 7. WRS spectrum (thin solid) of a single Langmuir-Blodgett layer of DMPE. The fitted curve (thick solid) is decomposed into the individual bands (dotted).

In this light, the decrease of the Raman intensity of this vibration in the monolayer spectrum can therefore indicate that the protein is more compactly folded with less amino acid residues exposed to the surrounding water molecules. Another possible explanation is that, since the protein is attached to the surface, only a small part of the protein can be in contact with water molecules. Although at this moment it is not exactly clear how the decrease of the Raman intensity of the $2931-\mathrm{cm}^{-1}$ band in the monolayer spectrum should be interpreted, it is evident that these measurements show the value of WRS in the study of protein conformational changes on adsorption at dielectric surfaces.

The result of the experiment on the LB monolayer is given in Figure 7. In order to excite the lipid molecules along their backbone, we used a TM0 mode, which is partly polarized perpendicular to the surface. The spectral region of the $\mathrm{C}-\mathrm{H}$ stretch vibrations from 2800 to $3000 \mathrm{~cm}^{-1}$ was recorded since it contains information about the degree of order in the lipid layer. The spectrum in this region, however, is very complicated due to the interaction between $\mathrm{C}-\mathrm{H}$ stretching fundamentals and overtones of the $\mathrm{CH}_{2}$ deformation modes. Therefore, the spectra were recorded with a spectral resolution of $1.5 \mathrm{~cm}^{-1} /$ pixel. The integration time was $10 \mathrm{~min}$, and the signal level was about 2 counts/s. The symmetric $\mathrm{C}-\mathrm{H}$ stretching mode at $2852 \mathrm{~cm}^{-1}$ is the fundamental d $\mathrm{d}^{+}$mode. ${ }^{22}$ Two bands at 2909 and $2936 \mathrm{~cm}^{-1}$ are due to Fermi resonance between the $\mathrm{d}^{+}$ fundamental and overtones of the $\mathrm{CH}_{2}$ deformation modes, which makes the fundamental mode sensitive to the lateral chain packing. ${ }^{22}$ The asymmetric $\mathrm{C}-\mathrm{H}$ stretch mode $\mathrm{d}^{-}$at 2882 is insensitive to the lateral packing, because the Fermi resonance interactions are symmetry forbidden, but is sensitive to the chain conformation. The width of this band increases if the conformation changes from trans to gauche. ${ }^{23}$ Therefore, the peak intensity ratio $I_{2882} / I_{2852}$ is sensitive to both lateral packing and conformation of the hydrocarbon chain. This ratio can range from 0.7 for completely melted hydrocarbon chains to 1.5 for vibrationally decoupled all-trans chains. ${ }^{25}$ We analyzed the monolayer spectrum by curve fitting with Lorentzian band shapes. The result of this fit is also shown in Figure 7 together with the individual bands. In Table 1, the fit parameters are given together with the assignments of the various Raman bands. The peak intensity ratio $I_{2882} / I_{2852}$ as determined from the fit has a value of 0.96 which corresponds to values reported ${ }^{10}$ for 
TABLE 1: Fit Results of the Spectra Given in Figure 7

\begin{tabular}{cccl}
\hline $\begin{array}{c}\text { wave- } \\
\text { numbers/ } \\
\mathrm{cm}^{-1}\end{array}$ & $\begin{array}{c}\text { intensity/ } \\
\text { au }\end{array}$ & $\begin{array}{c}\text { width/ } \\
\mathrm{cm}^{-1}\end{array}$ & \multicolumn{1}{c}{ assignment } \\
\hline 2852 & 46 & 20 & symm. C-H stretch $\mathrm{d}^{+}$of methylene \\
2882 & 44 & 35 & asymm. C-H stretch $\mathrm{d}^{-}$of methylene \\
2909 & 23 & 28 & Fermi resonance \\
2936 & 24 & 33 & Fermi resonance \\
2968 & 19 & 17 & in-plane asymm. C-H stretch of $\mathrm{CH}_{3}{ }^{b}$
\end{tabular}

${ }^{a}$ Reference 22. ${ }^{b}$ Reference 24 .

a CdA monolayer. The heat produced by the laser beam in the waveguide leads to a temperature increase which can melt the LB lipid layer. By comparing the Stokes to anti-Stokes intensity ratio of the $\mathrm{ZnO}$ vibration at $440 \mathrm{~cm}^{-1}$, the temperature can be determined and is not higher than approximately $50{ }^{\circ} \mathrm{C}$ at the used laser power. This is well below the melting temperature, which is about $100{ }^{\circ} \mathrm{C}$ for LB layers of lipids. It is argued ${ }^{10}$ that for a monolayer, the low value of the intensity ratio $I_{2882} /$ $I_{2852}$ is probably due to a high number of static gauche defects caused by substrate surface roughness and/or mismatch of the lattice parameter of the $\mathrm{LB}$ film with the $\mathrm{ZnO}$ surface.

\section{Conclusions}

By using a high refractive index waveguide with a thickness as small as $110-130 \mathrm{~nm}$, we showed that it is possible to obtain Raman spectra of very thin layers of polymers and monolayers of biological molecules without the use of resonant enhancement techniques. We expect that the use of WRS with $\mathrm{ZnO}$ waveguides can result in a useful tool to study thin-layer and monolayer assemblies on dielectric surfaces. Although the results obtained with the thin $\mathrm{ZnO}$ waveguides are very promising, the $\mathrm{ZnO}$ waveguide has two strong vibrations which can hamper the detection of weak vibrations of a monolayer around 440 and $1150 \mathrm{~cm}^{-1}$. Therefore, other waveguide materials with even better properties should be searched for.

Acknowledgment. We acknowledge the support provided to one of us (J. S. Kanger) by the S.T.W., Technology Foundation, Grant TTN 11.2511. We thank ir. C. E. H. Berger for preparing the LB films.

\section{References and Notes}

(1) Rabolt, J. F.; Swalen, J. D. In Spectroscopy of Surfaces. In Advances in Spectroscopy; Clark, R. J. H., Hester, R. E., Eds.; Wiley: Chichester, 1988; Vol. 16, Chapter 1, pp 1-36.

(2) Levy, Y.; Imbert, C.; Cipriani, S.; Racine, S.; Dupeyrat, R. Opt. Commun. 1974, 11, 11. 517.

(3) Rabolt, J. F.; Santo, R.; Swalen, J. D. Appl. Spectrosc. 1980, 34,

(4) Rabe, J. P.; Swalen, J. D.; Rabolt, J. F. Chem. Phys. 1987, 86, 1601.

(5) Rabe, J. P.; Novotny, V.; Swalen, J. D.; Rabolt, J. F. Thin Solid Films 1988, 159, 359.

(6) Ellahi, S.; Hester, r. E. Analyst 1994, 119, 491.

(7) Walker, D. S.; Hellinga, H. W.; Saavedra, S. S.; Reichert, W. M. J. Phys. Chem. 1993, 97, 10217. 549.

(8) Rabolt, J. F.; Santo, R.; Swalen, J. D. Appl. Spectrosc. 1979, 33,

(9) Rabolt, J. F.; Schlotter, N. E.; Swalen, R.; Santo, R. J. Polym. Sci.: Polym. Phys. Educ. 1983, 21, 1.

(10) Dierker, S. B.; Murray, C. A.; Legrange, J. D.; Schlotter, N. E. Chem. Phys. Lett. 1987, 137, 453.

(11) Schlotter, N. E. SPIE 1987, 822, 110.

(12) Kawai, T.; Umemura, J.; Takenaka, T. Chem. Phys. Lett. 1989, 162, 243.

(13) Knoll, W.; Philpott, M. R.; Swalen, J. D.; Girlando, A. J. Chem. Phys. 1982, 77, 2255.

(14) Duschl, C.; Knoll, W. J. Chem. Phys. 1988, 88, 4062.

(15) Ghatak, A. K.; Thyagarajan, K.; Shenoy, M. R. J. Lightwave Technol. 1987, LT-5, 660.

(16) Internal report.

(17) Soderquist, M. E.; Walton, A. G. J. Colloid Interface Sci. 1980, 75 (2), 386.

(18) Krisdhasima, V.; Vinarapong, P.; McGuire, J. J. Colloid Interface Sci. 1993, 161, 325.

(19) Schachar, R. A.; Solin, S. A. Invest. Ophtal. 1975, 14, 380.

(20) Yu, N. T.; East, E. J. J. Biol. Chem. 1975, 250, 2196.

(21) Verma, S. P.; Wallach, D. F. H. Biochem. Biophys. Res. Commun. 1977, 74, 473.

(22) Snyder, R. G.; Strauss, H. L.; Elliger, C. A. J. Phys. Chem. 1982, 86,5145 .

(23) Abbate, S.; Zerbi, G.; Wunder, S. L. J. Phys. Chem. 1982, 86, 3140.

(24) Sunder, S.; Mendelsohn, R.; Bernstein, H. J. Chem. Phys. Lipids 1976, $17,456$. 260.

(25) Gaber, B. P.; Peticolas, W. L. Biochim. Biophys. Acta 1977, 465, 